\title{
SLEEPexpert App - A Mobile Application to Support Insomnia Treatment for Patients with Severe Psychiatric Disorders
}

\author{
Kerstin DENECKE ${ }^{\mathrm{a}, 1}$, Carlotta L. SCHNEIDER ${ }^{\mathrm{b}}$, \\ Elisabeth HERTENSTEIN ${ }^{\mathrm{b}}$ and Christoph NISSEN ${ }^{\mathrm{b}}$ \\ a Bern University of Applied Sciences, Biel, Switzerland \\ ${ }^{\mathrm{b}}$ University Hospital of Psychiatry and Psychotherapy, University of Bern, Switzerland
}

\begin{abstract}
Cognitive behavior therapy for insomnia (CBT-I) is the first-line treatment for patients with insomnia disorder, including patients with severe mental disorders and comorbid insomnia. However, CBT-I is not sufficiently implemented in acute psychiatry settings. To make this treatment more accessible, we are currently adapting CBT-I to the needs of patients with severe psychiatric disorders in the form of a treatment program entitled SLEEPexpert. A core element of SLEEPexpert is keeping a sleep diary and restricting time in bed to increase sleep pressure. Here, we present a mobile application which supports the implementation of SLEEPexpert. The app is kept very simple, specifically designed for the target user group, and offers four main functionalities: entering information into the sleep diary, calculating the sleep efficiency and adapting the sleep window, delivering information on sleep and sleep disorders and accessing the recorded data in the sleep diary. Currently, we are preparing a usability test for the app aiming at fixing usability issues before running a clinical trial to assess the efficacy of this mHealth intervention.
\end{abstract}

Keywords. mHealth, sleep disorders, insomnia, behavioral therapy

\section{Introduction}

Mental disorders are highly prevalent with a lifetime-prevalence of about one fourth of the population and lead to significantly reduced quality of life worldwide. Insomnia, i.e. persistent difficulties falling and staying asleep, is very common in patients with mental disorders [1]. Insomnia is diagnosed based on disturbed sleep continuity and associated daytime impairment such as tiredness or reduced concentration reported by the patient. According to current guidelines, the first-line treatment is Cognitive Behavioral Therapy for Insomnia (CBT-I) [2]. Many patients with severe mental disorders suffer from cognitive impairment, reduced motivation and disorganized behavior, which often complicates the implementation of CBT-I. For this reason, we recently developed a pragmatic behavioral treatment program based on CBT-I ("Become your own SLEEPexpert") [3]. This program focuses on bedtime restriction as the most effective component of CBT-I [4]. SLEEPexpert uses a simplified sleep diary that can

\footnotetext{
${ }^{1}$ Corresponding Author, Kerstin Denecke, Bern University of Applied Sciences, Institute for Medical Informatics, Quellgasse 21, 2501 Biel / Switzerland; E-mail: kerstin.denecke@bfh.ch.
} 
offer valuable information about the individuals' sleep-wake pattern [5]. Sleep diaries have been established as a gold standard for subjective sleep assessment [6]. Although formats of those sleep diaries vary, they collect bed and sleep times and information on sleep satisfaction. The sleep diary forms the basis for bedtime restriction therapy [7] by providing the therapist with the information needed to restrict the bedtime to the actual total sleep time (TST) [8]. The diary should be continued throughout therapy for adapting the bedtime restriction when applicable. It serves as a self-monitoring tool, and may help the patients with insomnia to provide a more accurate picture of night-to-night sleep variation compared to a retrospective questionnaire. It may even offer relief to the individual by showing variation in sleep satisfaction [9], opposing to the common (mis)perception of persistent poor sleep quality in patients with insomnia. In addition, the sleep diary can be used to verify or falsify the common belief that sleep and wake quality are absolutely dependent, i.e. "bad" nights are always followed by a "bad" day.

The treatment program SLEEPexpert was developed to suit the needs of patients with severe psychiatric disorders. It combines an app-assisted behavioral intervention with face-to-face support and consists of three phases (therapist-guided treatment initiation, self-management with nurse support, and self-management). The mobile application aims at making this reduced CBT-I more accessible. In contrast to existing CBT-I apps, the app is tailored to the specific user group. As part of the treatment concept, it will enable patients with mental disorders to become their own sleep experts, i.e. be able to manage their sleep problems. In this paper, we describe the development of the app and its functionalities.

\section{Methods}

The application was developed in two phases in close collaboration with psychologists, medical doctors, nurses and patients. They accompanied the entire development process and provided feedback on mockup and prototype. In a first phase, requirements were collected by interviewing 3 experts. Based on these requirements a mockup was generated with Axure RP. The mockup was tested with patients hospitalized in a psychiatric ward to collect their feedback. This usability test with the mockup aimed at determining usability issues before the actual programming started and at collecting feedback on the interaction with the app, design issues and functionalities. The usability test was task-based, i.e. the participants had to solve a given task with the app and were asked to answer a questionnaire afterwards. The four tasks included: try to get information on sleep in general, get an overview on individual sleep behavior of the last days, have a look at the exercises, create a sleep diary entry. The questionnaire comprised the following five statements for which one option from a 5-item Likert scale (1=totally disagree, 5 = totally agree) had to be selected after solving a task:

- I could quickly solve the task.

- I was able to find the function quickly.

- Accessing the function is well designed.

- The functionality's result is as expected.

- The functionality is convenient.

Additionally, the participants were asked to suggest additional functionalities. The results from the usability test were considered for developing the final design and 
selecting the functionalities of the app. During the second phase, a prototype was implemented with the programming language Kotlin (https://kotlinlang.org). Data collected by the app is stored in a Google Firebase.

\section{Results}

\subsection{Requirements}

Our aim is to provide a digital application reflecting the pragmatic behavioral treatment program ("Become your own SLEEPexpert") customized to the needs of patients with acute psychiatric disorders and insomnia within a psychiatry setting. This means the application should be very simple with respect to data entry, but also regarding the suggestions for changing sleep behavior. The application should be attractive in using, e.g. by providing an intuitive visualization of the personal progress. It should motivate the patients to increase the number of entries in the sleep diary. The app has to support the collection of data on sleep behavior as it is relevant to support the treatment. The app has to be integrated in the three-step approach of the treatment program, i.e. it should be possible to use the app during the stay in the clinic, but also to continue using it afterwards in the personal environment to ensure a long-term improvement of the individual sleep behavior. Thus, it should encourage patients to maintain the newly acquired habits and should enable them to deal with their sleep problems in the long term. The app has to coach and educate the patient with respect the sleep disorders and has to provide means to improve the sleep behavior.

\subsection{Usability test results}

Based on these requirements, a mockup was created that allowed to keep a sleep diary and to access educational content. The usability test with the mockup was conducted with four patients. All patients were hospitalized in a psychiatric clinic at the time of the test. They were able to solve all given tasks in a short amount of time. For all tasks and questions, the value 4 (rather agree) was selected by all four participants. As additional functionalities, the participants suggested to include an alarm clock, music for falling asleep, the possibility to add comments in the diary, integrate with a mobile sensor such as Fitbit, and enabling voice input for the diary. Out of these suggestions, we included the alarm clock and the commenting option for the diary in the final version of the app. Since Android mobile phones provide the possibility to dictate, we resisted on implementing a voice user interface. The other suggestions were not integrated to avoid an overload with functionalities and limit complexity of user interfaces. It is to note that the target user group are patients with acute psychiatric disorders with varying health literacy, mental capacity and educational background.

\subsection{SLEEPexpert App}

The implemented prototype of the SLEEPexpert app provides the following functionalities: 1) Keeping a sleep diary, 2) Providing exercises and information on sleep, 3) Showing the progress on improving sleep behavior, 4) Alarm clock (see Figure 1). A surfer was used as a metaphor to illustrate sleep pressure and circadian variation. 
Like a surfer who has to wait for a big wave to build up, patients have to wait for sleep pressure to build up (e.g., should not go to bed too early). In addition, the correct time is critical for both surfing and sleeping (whether conditions for surfing, circadian type for sleep.

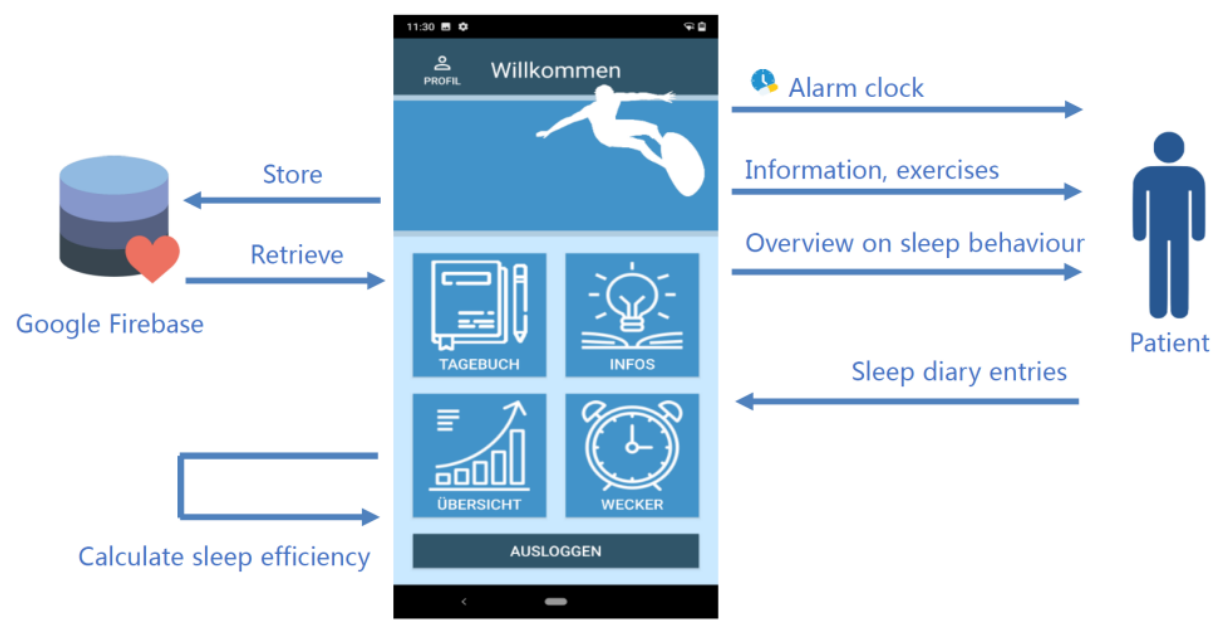

Figure 1. SLEEPexpert app: The home screen provides access to the four functionalities: diary, information, progress bar, alarm clock. The diary entry page (Tagebuch) asks for a judgement of the sleep quality on a scale of 1 to 8, for entering the time a person went to bed, got up and an estimation of the time a person slept

The sleep diary enables data entries when a person went to bed, when he or she got up in the morning, how long a person slept and asks for a judgement of the sleep quality. During the onboarding process, the user is asked to set an initial sleep window. There are two options: people who go to bed early and wake up early in the morning and those who go to sleep late at night and get up later in the morning. This initially selected sleep window is adapted automatically when a sufficient number of diary entries has been made. Sleep efficiency (percentage of time in bed that is actually spent asleep) is calculated by considering the last three entries in the sleep diary considering sleep time and time spent in bed. The value is only updated when data from 3 consecutive days is available. A progress bar shows the number of hours a person slept per night over time. The values are taken from the diary entries. When started for the first time, the user is informed on the functionalities of the SLEEPexpert app by a guided tutorial. The starting screen gives access to the main functionalities through four buttons. The number of functionalities is kept at a minimum to address the specific needs or backgrounds of the target user group. In this way, the interaction with the app can be kept as simple as possible. In its current implementation, the app is only running on Android. To store the data in the Google Firebase, an internet connection is required.

\section{Discussion and Future Work}

While several apps that deliver CBT-I are already available in English (e.g. Sleepio, CBT-I Coach), there is still no app available that targets at supporting users with acute psychiatric disorders in a psychiatric setting. Lyla Sleep coach and CBT-I Coach allow to keep a sleep diary and provide access to relaxation exercises. Lyla offers a six week 
program for better sleep and is available in Dutch. Sleepio offers a virtual character that serves as a coach who delivers weekly therapy sessions. Additionally, a sleep diary can be kept. In contrast to those apps, the SLEEPexpert app is characterized by its simplicity; it limits the requested data entries and interactions with the app. Furthermore, it is integrated in an entire treatment program that starts in the clinic with therapist-guided treatment initiation, followed by self-management with nurse support, and continues with self-management at home. SLEEPexpert focuses on the patients becoming "their own SLEEPexpert". This strategy is specifically important for patients with severe psychiatric disorders - the objective is to bring them into the position in taking over the responsibility for their own sleep behavior with support of the app. This might be of particular relevance in providing an alternative treatment approach to the frequent overprescription and over-use of hypnotics. A usability test with the app still has to be conducted for ensuring easy handling. Furthermore, data security and privacy has to be considered. Currently, the data is stored in a Google Firebase. Only a nick name and a password is used for patient identification. For a use in practice, the data could be stored in a health bank like MIDATA (https://www.midata.coop/) using FHIR. Such health bank ensures data privacy and security. This would also enable patients to give researchers their consent for using the data for studying the efficacy of the SLEEPexpert treatment concept in psychiatric wards. A web platform would be useful for therapists to access the data collected by their patients and as a basis to discuss follow-up treatment with the patient. We already started to design extensions of the application. A quiz will allow users to test and train their knowledge related to sleep, healthy behaviour and insomnia.

\section{Acknowledgements}

We thank Andi Ademi, Luca Leuenberger, Andy Landolt, Janahan Sellathurai and Sugeelan Selvasingham for implementing the app as part of their semester project.

\section{References}

[1] Bei B, Asarnow LD, Krystal A, Edinger JD, Buysse DJ, Manber R. Treating insomnia in depression: Insomnia related factors predict long-term depression trajectories. J Consult Clin Psychol. 2018;86(3):282-293. doi:10.1037/ccp0000282

[2] Riemann D, Baglioni C, Bassetti C, et al. European guideline for the diagnosis and treatment of insomnia. J Sleep Res. 2017;26(6):675-700. doi:10.1111/jsr.12594

[3] Schneider C, Hertenstein E, Fehér K, et al. Become your own SLEEPexpert: design, implementation and first evaluation of a pragmatic behavioral treatment program for insomnia in patients with acute psychiatric disorders. Sleep Advances. 2020. Major Revision.

[4] Miller CB, Espie CA, Epstein DR, et al. The evidence base of sleep restriction therapy for treating insomnia disorder. Sleep Med Rev. 2014;18(5):415-424. doi:10.1016/j.smrv.2014.01.006

[5] Riemann D, Baglioni C, Bassetti C, et al. European guideline for the diagnosis and treatment of insomnia. J Sleep Res. 2017;26(6):675-700. doi:10.1111/jsr.12594

[6] Carney CE, Buysse DJ, Ancoli-Israel S, Edinger JD, Krystal AD, Lichstein KL, Morin CM. The consensus sleep diary: standardizing prospective sleep self-monitoring. SLEEP 2012;35(2):287-302.

[7] Troxel, WM, Germain A, Buysse DJ. Clinical management of insomnia with brief behavioral treatment (BBTI). Behav Sleep Med. 2012,;10: 266-279. doi: 10.1080/15402002.2011.607200

[8] Hertenstein E, Nissen C, Riemann D. Pharmacological and Non-Pharmacological Treatments of Insomnia. Vol 1. Oxford University Press; 2017.

[9] Hertenstein E, Spiegelhalder K, Johann A, Riemann D. Prävention und Psychotherapie der Insomnie in Störungsspezifische Psychotherapie. Verlag W. Kohlhammer; 2015: 30-31. 